\title{
Induction of labour versus conservative management for mild gestational hypertension at term
}

\author{
Pallipuram S. Bhageerathy*, Vinotha Thomas, Annie Regi, Ruby Jose
}

Department of Obstetrics and Gynaecology, Christian Medical College, Vellore, Tamil Nadu, India

Received: 31 December 2015

Revised: 23 January 2016

Accepted: 08 February 2016

\section{*Correspondence:}

Dr. Pallipuram S. Bhageerathy,

E-mail: drbhagee@gmail.com

Copyright: ( $)$ the author(s), publisher and licensee Medip Academy. This is an open-access article distributed under the terms of the Creative Commons Attribution Non-Commercial License, which permits unrestricted non-commercial use, distribution, and reproduction in any medium, provided the original work is properly cited.

\begin{abstract}
Background: Hypertensive disorders of pregnancy remain a leading cause of maternal and perinatal morbidity and mortality. The aim of this study was to find out whether immediate induction of labour in women with singleton pregnancy complicated by mild gestational hypertension at term reduced maternal and neonatal morbidity, mortality and expenditure when compared to conservative management without increasing instrumental delivery and caesarean section rates.

Methods: This was a randomized controlled trial to compare immediate induction of labour to conservative management for mild gestational hypertension in women between 18-35 years of age with singleton pregnancy with mild gestational hypertension at 37-39.5 weeks without any other complications. Eligible patients presenting to the obstetric outpatient department or labour room of Christian Medical College, Vellore with gestational hypertension were randomized (49 patients in the induction arm and 51 patients in the conservative arm) and followed up. The maternal and neonatal outcomes in both groups were compared.

Results: There was no maternal mortality in both the groups. There was increased incidence of composite maternal morbidity (pre-eclampsia, eclampsia, HELLP syndrome, pulmonary oedema, renal failure, thromboembolic disease, abruption, need for ICU care and major postpartum haemorrhage) in the conservative arm when compared to induction arm (14 versus 8), though not statistically significant (p 0.23). There was no significant difference in the caesarean section rates between the two groups (p 0.313 and 0.306 respectively) despite the much favorable Bishop score in the conservative group ( $\mathrm{p}$ 0.054). There was no significant difference in neonatal morbidity and mortality. A slight increase by about 600 rupees in the median total cost was found with conservative management when compared to induction group.

Conclusions: The study did not show a statistically significant difference in maternal mortality, composite maternal morbidity, neonatal mortality and morbidity as well as treatment cost between immediate induction of labour and conservative management for mild gestational hypertension at term.
\end{abstract}

Keywords: Induction of labour, Mild gestational hypertension, Expectant management, Term

\section{INTRODUCTION}

Hypertensive disorders of pregnancy remain a leading cause of maternal and perinatal morbidity and mortality. The management of severe gestational hypertension and preeclampsia at term is clear. The only definitive treatment for the same is delivery. ${ }^{1}$ But the management of mild gestational hypertension without any other complication at term is not clear. Induction of labour is thought to prevent the progression of hypertension and its complications like eclampsia, HELLP syndrome, placental abruption, renal failure etc. but doing so could increase the instrumental and caesarean delivery rates. The HYPITAT trial done on 756 patients with gestational 
hypertension or mild pre-eclampsia after 36 weeks from Netherlands concluded that induction of labour improved maternal outcome. ${ }^{2}$ But there is paucity of data for the management of mild gestational hypertension at term from Indian population.

\section{Aims and objectives}

1. To study whether immediate induction of labour in women with singleton pregnancy complicated by mild gestational hypertension at 37 weeks reduced maternal and neonatal morbidity and mortality when compared to conservative management.

2. To study whether immediate induction of labour increased the instrumental and caesarean delivery rates.

3. To study whether immediate induction of labour would be cost effective when compared to conservative management as the costs for foetal surveillance and increased number of antenatal visits could be avoided.

\section{METHODS}

All women with a singleton pregnancy, aged 18 to 35 years, with cephalic presentation at 37 to 40 weeks of gestation, with mild gestational hypertension were eligible for inclusion into the study. Only those with a systolic BP between 140 and $159 \mathrm{~mm}$ of $\mathrm{Hg}$ and a diastolic BP between 90 and $100 \mathrm{~mm}$ of $\mathrm{Hg}$ (Korotkoff Phase V) repeated after 4 hours were recruited. Women with proteinuria were excluded from the study, as also those with pre-eclampsia, severe gestational hypertension, chronic hypertension, Gestational Diabetes Mellitus, renal disease, heart disease and previous caesarean delivery.

Women carrying growth restricted foetuses (less than 2.5 $\mathrm{kg}$ ) were excluded from the study. If foetal compromise by way of oligohydramnios on ultrasound scan or nonreassuring foetal heart status on non-stress test was present, or if there were foetal anomalies, those women were excluded from the study.

Randomization was done by computer generated randomization codes using the software RALLOC. Sealed opaque envelopes were used for allocation concealment. Blinding and masking of intervention allocation was not possible.

\section{Methodology}

Eligible patients presenting to the Obstetric outpatient department (OPD) or labour room of Christian Medical College, Vellore from September 2012 to August 2014 were randomized in a $1: 1$ ratio to receive immediate induction of labour (group 1) or conservative management (group 2) after taking informed written consent.
For those in the immediate induction arm (group 1), a vaginal examination was done to assess the Bishop's score. If the score was 6 or more, artificial rupture of membranes with or without oxytocin augmentation was done within 12 hours of randomization. If the score was less than 6 , cervical ripening was done with $\mathrm{PGE}_{1}(25$ microgram $6^{\text {th }}$ hourly for 2 doses) as is the routine for induction of labour in our hospital.

For those allocated to the conservative management arm (group 2), pregnancy induced hypertension (PIH) work up which included platelet count, serum creatinine, serum transaminases (SGOT, SGPT), lactate dehydrogenase (LDH) and blood picture was done. They were advised daily home blood pressure (BP) monitoring by a local doctor or nurse who recorded it. Biweekly visit to the outpatient department was advised until they went into spontaneous labour or till 39 weeks and 5 days when labour was induced as mentioned above. If there was progression of the disease by way of increase in diastolic $\mathrm{BP}$ to $100 \mathrm{~mm}$ of $\mathrm{Hg}$ or more, or proteinuria became more than or equal to $1+$ by dipstick method, or if the patient developed signs and symptoms of impending eclampsia, suspected foetal distress, eclampsia or HELLP syndrome, labour was induced according to the protocol for induction of labour.

The demographic details, details of delivery, drugs used, intrapartum and postnatal complications for the mother and the baby were noted.

The primary outcomes measured for the mother were maternal mortality and composite maternal morbidity (pre-eclampsia, eclampsia, HELLP syndrome, pulmonary oedema, renal failure, thromboembolic disease, abruption, need for ICU care and major postpartum haemorrhage). The primary outcome measured for the baby was perinatal mortality.

The secondary outcomes measured for the mother was the mode of delivery (normal/ instrumental/ caesarean), need for anticonvulsant, need for antihypertensive drugs (intrapartum/postnatal). The secondary outcomes measured for the baby were neonatal morbidity ( 5 minute Apgar score less than 7, cord $\mathrm{pH}$ less than 7, admission to neonatal intensive care unit).

The cost analysis was done only for the direct medical costs. This was done by adding the inpatient medical bill of the mother and the baby along with the extra consultation charges and the charges for the tests for maternal and foetal well-being for patients on conservative management and compared with the final medical inpatient bill of the mother and the baby at discharge for the patients in the induction group.

\section{Statistical analysis}

Chi square test was used for test of categorical variables. Student's t test was used for test of maternal morbidity 
and comparison of continuous variables. Treatment effect was presented as relative risk with $95 \%$ confidence interval. Stratified analysis was done using logistic regression, presenting as odd's ratio for primary outcome. A $p$ value of less than 0.05 indicates statistical significance.

\section{RESULTS}

During the study period, 624 women with mild gestational hypertension were assessed for eligibility for the trial. Of these, 509 women were excluded as they did not meet the inclusion criteria. Thirteen patients who fulfilled the inclusion criteria did not give consent. A total of 102 patients were randomised to the trial of which 50 were allocated to the induction of labour arm and 52 were allocated to the conservative management arm. In the induction group, one patient refused induction of labour after randomisation and one in the conservative management group was lost to follow up. The patient profile of the study is depicted in a flowchart in Figure 1.

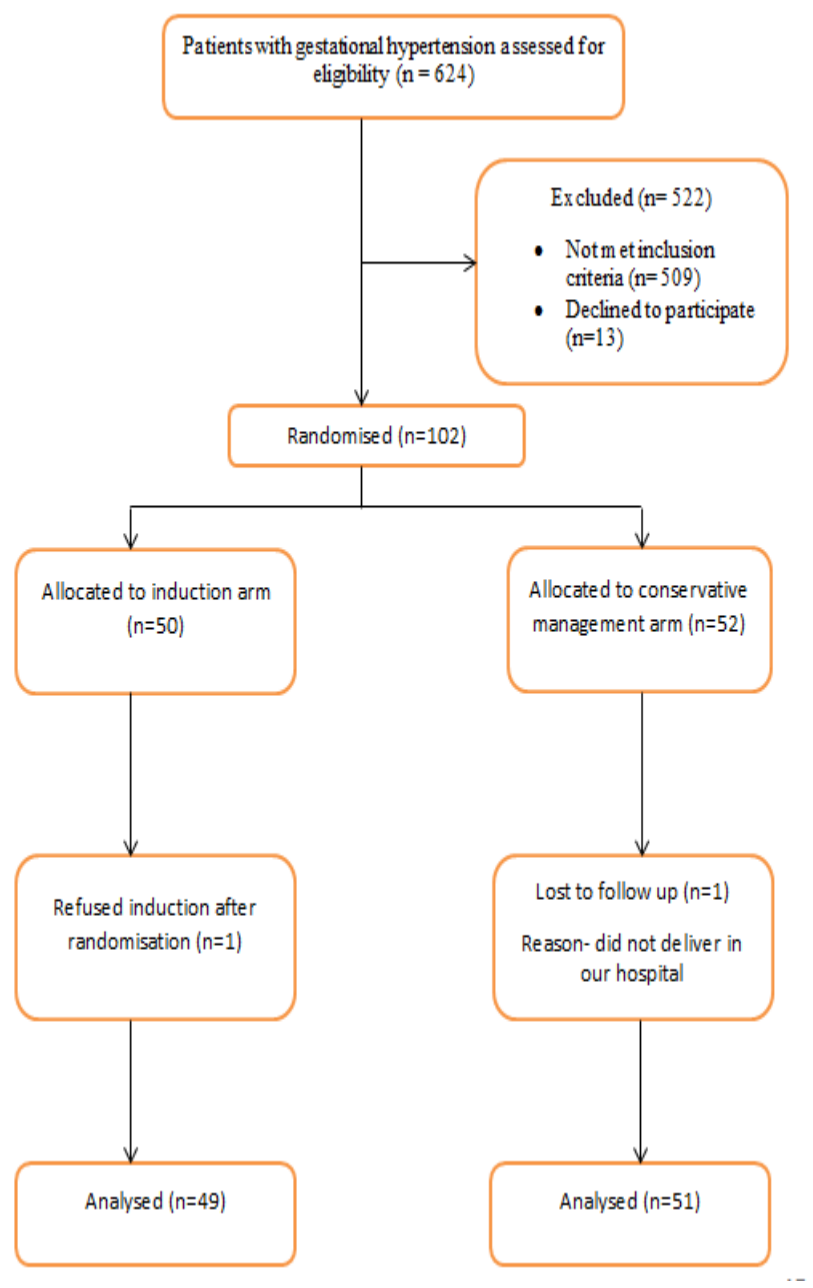

Figure 1: Flowchart showing the patient profile.
The baseline characteristics like maternal age, body mass index, level of education, gestational age at randomization, average systolic and diastolic blood pressures were comparable in both groups of study women (Table 1). There were 40 primigravidae and 9 multigravidae in the induction arm and 38 primigravidae and 13 multigravidae in the conservative arm. The average gestational age at delivery was 38 weeks in the induction arm and 39 weeks in the conservative management arm.

Regarding the primary outcome of maternal mortality, there was no maternal mortality in both the groups.

There was increased incidence of composite maternal morbidity (pre-eclampsia, eclampsia, HELLP syndrome, pulmonary oedema, renal failure, thromboembolic disease, abruption, need for ICU care and major postpartum haemorrhage) in the conservative management arm when compared to the induction arm. In the conservative group, 14 patients (27.5\%) had composite maternal morbidity when compared to 8 patients $(16.8 \%)$ in the immediate induction group, though it was not statistically significant (p 0.23). When the complications were considered individually, progression to severe hypertension and pre-eclampsia was increased in the conservative group when compared to the immediate induction group, though not statistically significant. In the conservative group, four patients (7.8\%) progressed to severe hypertension when compared to one patient $(2 \%)$ in the induction group (p 0.363). Eighteen patients in the conservative group progressed to pre-eclampsia, 5 to mild and 13 to severe pre-eclampsia whereas only 10 patients in the induction group progressed to preeclampsia, 4 to mild and 6 to severe preeclampsia (p 0.677).

Surprisingly, one patient in the immediate induction arm had intrapartum eclampsia while none in the conservative arm had eclampsia. None of the patients in either group had complications like HELLP syndrome, pulmonary oedema, renal failure or placental abruption and required ICU admission. Two percentage of women in the induction group and $3.9 \%$ in the conservative group had postpartum haemorrhage which was managed medically (p 1.0). None of them required blood transfusion. One patient in the conservative management arm, who delivered normally at 39 weeks and 5 days and was discharged, got readmitted with cortical vein thrombosis on her $12^{\text {th }}$ postpartum day.

When considering the primary outcomes for the foetus and neonate, there was only 1 neonatal death in the induction group due to perinatal asphyxia but this was not statistically significant (p 0.490). There were no intrauterine deaths in either group. 
Table 1: Baseline characteristics.

\begin{tabular}{|c|c|c|c|c|c|c|c|}
\hline \multirow[t]{2}{*}{ Baseline characteristics } & \multicolumn{3}{|l|}{ Group 1} & \multicolumn{4}{|c|}{ Group 2} \\
\hline & Mean (SD) & Minimum & Maximum & \multicolumn{2}{|c|}{ Mean (SD) } & Minimum & Maximum \\
\hline Gestational age at trial entry & $38.0(0.82)$ & 37.0 & 39.4 & \multicolumn{2}{|c|}{$37.97(0.65)$} & 37.0 & 39.2 \\
\hline Maternal Age & $25.37(3.91)$ & 19 & 35 & \multicolumn{2}{|c|}{$25.90(3.44)$} & 19 & 32 \\
\hline Systolic BP at trial entry & $143.6(4.78)$ & 140 & 156 & \multicolumn{2}{|c|}{$141.96(3.63)$} & 140 & 51 \\
\hline Diastolic BP at trial entry & $92.53(3.82)$ & 90 & 100 & 91. & 3.54) & 90 & 100 \\
\hline \multirow{2}{*}{ Level of Education } & \multicolumn{3}{|l|}{ Group 1} & \multicolumn{3}{|c|}{ Group 2} & \multirow{2}{*}{$\mathrm{p}$ value } \\
\hline & $\mathrm{N}$ & \multicolumn{2}{|l|}{$\%$} & $\mathrm{~N}$ & \multicolumn{2}{|l|}{$\%$} & \\
\hline Nil & 2 & \multicolumn{2}{|l|}{4.1} & 2 & \multicolumn{2}{|l|}{3.9} & \multirow{4}{*}{0.847} \\
\hline Primary & 27 & 55.1 & & 25 & 49.0 & & \\
\hline Secondary & 18 & 36.7 & & 20 & 39.2 & & \\
\hline Professional & 2 & 4.1 & & 4 & 7.8 & & \\
\hline \multicolumn{7}{|l|}{ Obstetric score } & \multirow{3}{*}{0.472} \\
\hline Primi & 40 & 81.6 & & 38 & 74.5 & & \\
\hline Multi & 9 & 18.4 & & 13 & 25.5 & & \\
\hline \multicolumn{7}{|l|}{ BMI } & \multirow{4}{*}{0.114} \\
\hline$<19$ & 4 & 8.2 & & - & - & & \\
\hline $19-24.9$ & 25 & 51.0 & & 28 & 54.9 & & \\
\hline $25-29.9$ & 20 & 40.8 & & 23 & 45.1 & & \\
\hline
\end{tabular}

Table 2: Indications for induction in the conservative management group.

\begin{tabular}{|c|c|c|c|}
\hline \multirow[t]{2}{*}{$\begin{array}{l}\text { Indication for } \\
\text { induction }\end{array}$} & \multicolumn{2}{|c|}{$\begin{array}{l}\text { Conservative } \\
\text { management } \\
\text { group }\end{array}$} & \multirow[t]{2}{*}{ p-value } \\
\hline & $\mathrm{n}$ & $\%$ & \\
\hline Pre-eclampsia & 11 & 26.2 & \multirow{5}{*}{0.815} \\
\hline PROM & 7 & 16.7 & \\
\hline Suspected foetal distress & 3 & 7.1 & \\
\hline 39 Weeks +5 days & 16 & 38.1 & \\
\hline Others & 5 & 11.9 & \\
\hline
\end{tabular}

\section{Secondary outcomes for the mother}

There were more patients with spontaneous onset of labour in the conservative management arm when compared to the induction arm (11 versus 3$)$. Three patients in the induction group who had a favourable Bishop score went into labour after vaginal examination and did not require oxytocin augmentation. Thus, 94\% of patients in the induction arm underwent induction of labour (3 went into spontaneous labour) whereas $78.4 \%$ of patients in the conservative management arm had to be induced for various reasons before 40 weeks ( $p$ 0.041). This was statistically significant. The indications for induction of labour in the conservative management group are shown in Table 2. Patients on conservative management were found to have a more favourable Bishop score at the onset of induction of labour though not statistically significant (p 0.054).

There was no statistically significant difference in the instrumental delivery and caesarean section rates between the two groups (p 0.313 and 0.306 respectively) as shown in Figure 2. This was despite a much favourable Bishop score in the conservative management group when compared to the induction group (0.054). Though there were more women who went into spontaneous onset of labour in the conservative management group, the caesarean section rate was almost the same in both groups. This may be because of a slightly higher rate of progression of the disease in the conservative management group which predisposed them to caesarean section.

Patients on conservative management required more intrapartum use of antihypertensive drugs (p 0.063) and

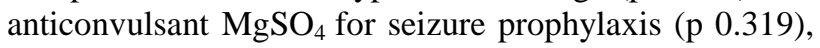
though not significant statistically.

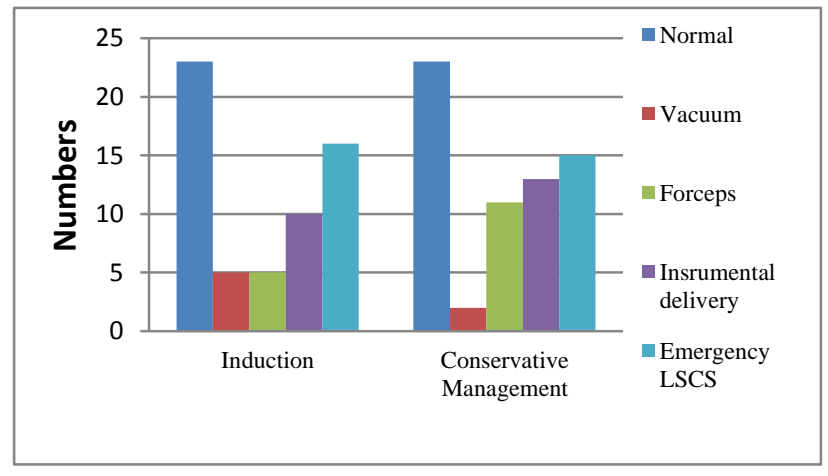

Figure 2: Mode of delivery.

\section{Secondary outcomes for the baby}

There was no statistically significant difference in the neonatal morbidity between the 2 groups. A slight 
increase in neonatal ICU admissions was found in the induction arm ( $\mathrm{p}$ 0.357). The mean birth weight in the induction arm was lower than that in the conservative $\operatorname{arm}(2.8 \mathrm{~kg}$ versus $3 \mathrm{~kg})$, though there was no statistically significant difference in the incidence of growth restricted babies between the 2 groups.

\section{Cost analysis}

A slight increase by about 600 rupees in the median total cost was found in the conservative management when compared to the induction group, though it was not significant statistically as shown in Figure 3. This may be attributable to the tests of maternal and foetal surveillance and the increased number of hospital visits in the patients on conservative management. The mean cost was not taken for statistical analysis because of the error in calculation due to the very high maximum cost in the induction group for 1 patient whose baby had perinatal asphyxia and was admitted in NICU for 6 days.

The results are summarised in Table 3 .

Table 3: Results.

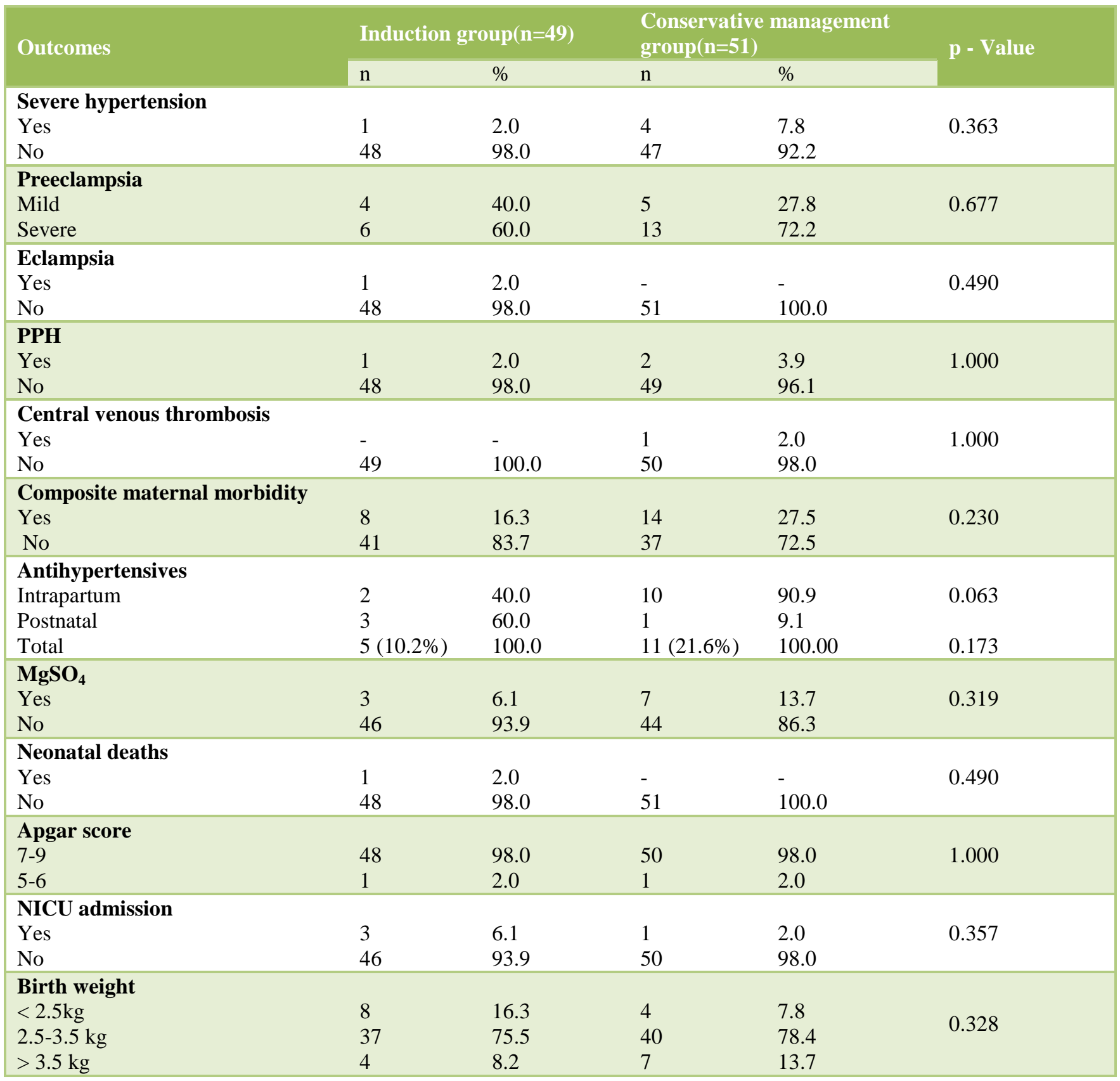




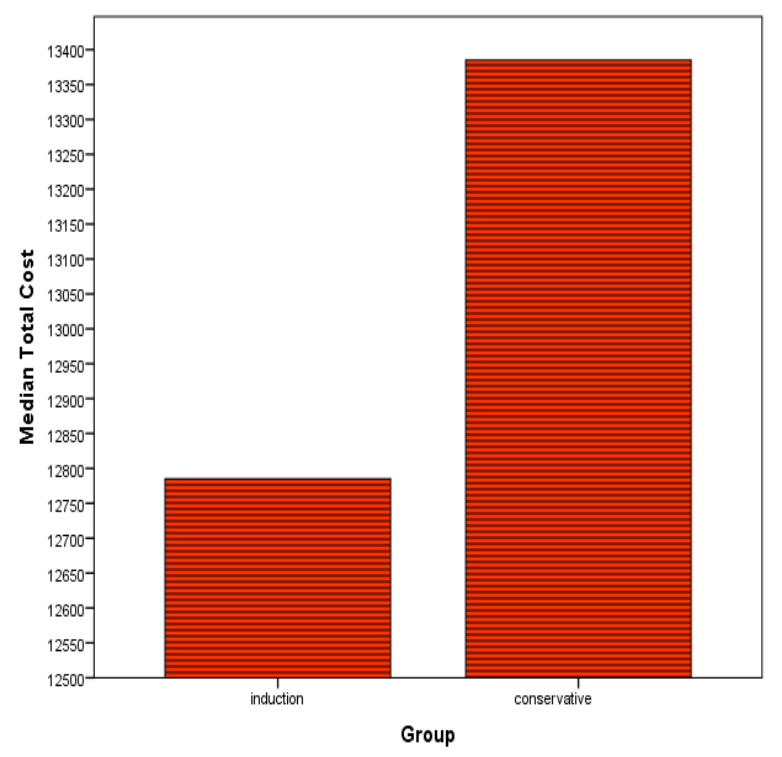

Figure 3: Cost analysis.

\section{DISCUSSION}

Hypertensive disorders complicate $5-10 \%$ of all pregnancies. ${ }^{3}$ It accounts for $9.1 \%$ and $16.1 \%$ of maternal deaths in developing and developed countries respectively4. In our hospital, mild gestational hypertension accounts for $2-4 \%$ of all pregnancies. It is found that about $10 \%$ of women with gestational hypertension at term progress to pre-eclampsia and its complications. $^{5}$

In a comparative study of 35,000 singleton pregnancies to investigate the impact of hypertensive disorders of pregnancy, Lau TK et al found that the incidence of growth restricted babies was significantly higher in subjects with pre-eclampsia and eclampsia when compared to the control group. In contrast, there was no significant difference in the incidence of growth restricted babies among gestational hypertensives when compared to normotensives. ${ }^{6}$ Hence there exists a dilemma as to whether women with mild gestational hypertension at term need to be induced or can be managed conservatively like normotensive women.

The present study was planned to answer the question of whether induction of labour was better in women with mild hypertensive disease of pregnancy without any other complication at term when compared to expectant management.

The HYPITAT (Induction of labour versus expectant monitoring for gestational hypertension or mild preeclampsia after 36 weeks' gestation) study proved that induction of labour was associated with improved maternal outcome and suggested that it should be the treatment of choice for women with mild hypertensive disease beyond 37 weeks $(\mathrm{p}<0.0001) .{ }^{2}$ Though the sample size in our study was not adequate to attain the desired power, the main primary outcomes of maternal and perinatal mortality were similar in both the arms of the study. The composite maternal morbidity and progression to severe hypertension was found to be increased in the conservative management group ( $p \quad 0.23$ and 0.36 respectively), though not statistically significant. Though our results show a similar trend of increasing maternal morbidity with conservative management, we did not get statistically significant results unlike the HYPITAT trial.

We did not find any statistically significant difference in the caesarean section rates between the 2 groups. Sixteen patients $(32.7 \%)$ in the induction group when compared to 15 patients $(29.4 \%)$ in the conservative management group had caesarean section (p 0.317) in our study. This was in spite of a much favourable Bishop score in the conservative management group when compared to the induction group, probably due to progression of the disease which made them more prone for caesarean section. This was different from the results of the HYPITAT study group according to which fewer caesarean sections were needed in the induction group when compared to expectant monitoring group which could be due to decreased occurrence of severe maternal morbidity in the induction group. ${ }^{7}$ They also found that induction of labour was more beneficial in those with an unfavourable cervix contrary to the popular belief. ${ }^{8}$ This beneficial effect of induction of labour was not found in women with gestational hypertension or mild preeclampsia between 36 and 37 weeks in subgroup analysis. ${ }^{9}$

The mean birth weight in the induction arm was lower than that in the conservative management arm $(2.8 \mathrm{~kg}$ versus $3 \mathrm{~kg}$ ) which was similar to the results from the HYPITAT study. ${ }^{7}$ But there was no statistically significant difference in the incidence of growth restricted or big babies between the 2 groups.

The cost analysis in our study showed that induction of labour was slightly cheaper than conservative management, though this was not significant statistically. An economic analysis of induction of labour and expectant monitoring in women with gestational hypertension or pre-eclampsia at term based on HYPITAT data showed that induction of labour was $11 \%$ cheaper than expectant management. ${ }^{10}$ The lack of statistical significance may be due to the fact that the sample size calculated based on the results of the HYPITAT study was 342, but only 100 patients could be finally analyzed in our study.

The impact of HYPITAT trial on doctors' behaviour was studied in Netherlands and it was found that there was an increase in induction of labour from 58.3 to $67.1 \%$ (p < $0.001)$ and decrease in prevalence of eclampsia from 0.85 to $0.19 \%(\mathrm{p}<0.001)$ before and after the trial. ${ }^{11}$ 


\section{CONCLUSIONS}

The study did not show a statistically significant difference in maternal mortality, composite maternal morbidity, expenditure, neonatal mortality and morbidity between induction of labour and conservative management for mild gestational hypertension at term. A larger study is recommended to show a significant benefit of induction of labour when compared to conservative management for mild gestational hypertension at term.

\section{ACKNOWLEDGEMENTS}

The authors are extremely thankful to Visalakshi and Tunny Sebastian from the Department of Biostatistics, Christian Medical College, Vellore for their help with the statistics. We are indebted to all the patients who consented to take part in the study.

Funding: Institutional Review Board, Christian Medical College, Vellore

Conflict of interest: None declared

Ethical approval: The study was approved by the Institutional Ethics Committee

\section{REFERENCES}

1. Ganzvoort W, Sibai BM. Temporising versus interventionist management (preterm and at term). Best Pract Res Clin Obstet Gynaecol. 2011;25(4):463-76.

2. Koopmans CM, Bijlenga D, Aarnoudse JG, van Beek E, Bekedam DJ, van den Berg PP et al. Induction of labour versus expectant monitoring in women with pregnancy induced hypertension or mild preclampsia at term: the HYPITAT trial. BMC Pregnancy Childbirth. 2007;7:14.

3. Duley L. The global impact of pre-eclampsia and eclampsia. Semin Perinatol. 2009;33:130-37.

4. Khan KS, Wojdyla D, Say L. WHO analysis of causes of maternal death: a systematic review. Lancet. 2006;365:1006-74.
5. Saudan P. Does gestational hypertension become pre-eclampsia? $\mathrm{Br} \quad \mathrm{J}$ Obstet Gynaecol. 1998;105:1177-84.

6. Lau TK, Pang MW, Sahota DS, Leung TN. Impact of hypertensive disorders of pregnancy at term on infant birth weight. Acta Obstet Gynecol Scand. 2005;84:875-7.

7. Koopmans CM, Bijlenga D, Groen H, Vijgen SM, Aarnoudse JG, Bekedam DJ et al. Induction of labour versus expectant monitoring for gestational hypertension or mild pre-eclampsia after 36 weeks' gestation (HYPITAT): a multicenter, open-label randomized controlled trial. Lancet. 2009;374:97988.

8. Tajik P, van der Tuuk K, Koopmans CM, Groen H, van Pampus MG, van der Berg PP et al. Should cervical favorability play a role in the decision for labour induction in gestational hypertension or mild preeclampsia at term? An exploratory analysis of the HYPITAT trial. $\mathrm{Br} \quad \mathrm{J}$ Obstet Gynaecol. 2012;119:1123-30.

9. Johnson DD. Induced labour for preeclampsia and gestational hypertension. Lancet. 2009;374:951-2.

10. Mortiary T. An economic analysis of induction of labour and expectant monitoring in women with gestational hypertension or preeclampsia at term (HYPITAT trial). $\mathrm{Br} \mathrm{J}$ Obstet Gynaecol. 2011;118:763.

11. vaan der Tuuk K, Koopmans CM, Groen H, Mol BW, van Pampus MG, HYPITAT study group. Impact of HYPITAT trial on doctors' behavior and prevalence of eclampsia in the Netherlands. $\mathrm{Br} \mathrm{J}$ Obstet Gynaecol. 2011;118:1658-60.

Cite this article as: Bhageerathy PS, Thomas V, Regi A, Jose R. Induction of labour versus conservative management for mild gestational hypertension at term. Int J Reprod Contracept Obstet Gynecol 2016;5:689-95. 